\title{
UM NOVO MÉTODO PARA O CÁLCULO DA PROBABILIDADE DE ERRO DE BIT DE ESQUEMAS DE MODULAÇÃO QAM SUJEITOS AO DESVANECIMENTO RAYLEIGH
}

\author{
Waslon Terllizzie Araújo Lopes, Francisco Madeiro e Marcelo Sampaio de Alencar
}

\begin{abstract}
Resumo - Neste artigo é apresentado um novo método para obtenção de expressões exatas para a probabilidade de erro de bit (BEP) de esquemas de modulação sujeitos ao desvanecimento Rayleigh. O método modela o canal com desvanecimento Rayleigh como um canal sujeito a um ruído aditivo, cuja distribuição de probabilidade é vista como resultado da razão entre uma variável aleatória (v.a.) gaussiana e uma v.a. Rayleigh. O método consiste em usar a função cumulativa de probabilidade unidimensional desse ruído aditivo para obter expressões fechadas para a BEP de esquemas de modulação QAM (quadrature amplitude modulation) sujeitos ao desvanecimento. Em particular, o método proposto é usado para obter expressões novas, exatas e fechadas para a BEP dos esquemas 16-QAM, 64-QAM e 256-QAM. São apresentados resultados de simulação que corroboram as expressões obtidas.
\end{abstract}

Palavras-chave: Comunicações móveis, canal com desvanecimento Rayleigh, probabilidade de erro de bit, QAM.

\begin{abstract}
This paper presents a new method for determining exact expressions for the bit error probability (BEP) of modulation schemes subject to Rayleigh fading. In this method the Rayleigh fading channel is seen as an additive noise channel whose noise is modeled as the ratio between a Gaussian random variable (r.v.) and a Rayleigh r.v. The method is based on the use of the cumulative density function of that additive noise to obtain closed-form expressions for the BEP of QAM (quadrature amplitude modulation) schemes, subject to fading. In particular, the proposed method is used to obtain new, exact and closed-form expressions for the BEP of 16-QAM, 64-QAM and 256-QAM. These expressions are shown to be in agreement with simulation results.
\end{abstract}

Keywords: Mobile communications, Rayleigh fading channel, bit error probability, QAM.

Waslon Terllizzie Araújo Lopes está no Departamento de Engenharia Elétrica, Faculdade de Ciência e Tecnologia (ÁREA1), Salvador, BA, Brasil. Francisco Madeiro Bernardino Junior está no Departamento de Estatística e Informática, Universidade Católica de Pernambuco, Recife, PE, Brasil. Marcelo Sampaio de Alencar está no Laboratório de Comunicações, Departamento de Engenharia Elétrica, Universidade Federal de Campina Grande, Campina Grande, PB, Brasil. E-mails: waslon@area1.br, madeiro@dei.unicap.br, malencar@dee.ufcg.edu.br. Editor de Área responsável: Ricardo Menezes Campello de Souza. Artigo submetido em 24/Fev/2003, revisado em 16/Abr/2004, aceito em 03/Jun/2004.

\section{INTRODUÇÃO}

A obtenção de taxas de transmissão elevadas, com confiabilidade, sujeitas à restrição de disponibilidade de um canal com largura de faixa muito limitada, constitui um dos grandes desafios em sistemas de comunicações sem fio. O canal móvel, por exemplo, apresenta eficiência espectral inferior ao seu limite teórico. O esquema QAM (quadrature amplitude modulation) é uma técnica de modulação atraente no que diz respeito à eficiência em termos de largura de faixa. Estudos (e.g. [1-6]) têm sido desenvolvidos no sentido de avaliar o desempenho de esquemas de modulação QAM em termos de probabilidade de erro de bit (BEP - Bit Error Probability). Nesse contexto, a obtenção de expressões fechadas para a BEP de esquemas QAM é um problema relevante em sistemas de comunicações.

Estudos relatados na literatura têm apresentado expressões para a BEP de esquemas QAM em um canal com ruído aditivo gaussiano branco (AWGN, additive white Gaussian noise). Convencionalmente, aproximações para a BEP de esquemas de modulação QAM têm sido obtidas calculando a probabilidade de erro de símbolo ou simplesmente estimando-a com o uso de limites inferiores ou superiores [7]. Aproximações melhores para a BEP de esquemas QAM sujeitos a AWGN foram apresentadas recentemente em [1,2]. É importante mencionar que, apesar de algumas expressões aproximadas apresentadas na literatura fornecerem valores com precisão ao ser considerada uma relação sinal-ruído (SNR, signal-to-noise ratio) elevada, a avaliação da BEP utilizando essas expressões tende a se afastar dos valores exatos correspondentes ao ser considerada uma SNR baixa. Apesar de muita atenção ter sido dedicada à avaliação de desempenho de QAM em termos de probabilidade de erro de bit, só recentemente, em uma contribuição de Cho e Yoon [8], foi obtida uma expressão exata, fechada, para a probabilidade de erro de bit do esquema QAM $M$-ário ( $M$-QAM) em um canal AWGN quando se usa um mapeamento de Gray.

Em se tratando da avaliação de desempenho de esquemas QAM em um canal com desvanecimento Rayleigh, a probabilidade de erro de bit tem sido objeto de interesse em alguns trabalhos, podendo ser citadas as contribuições de Fortune et al. [3,5], Shayesteh e Aghamohammadi [4] e Vitthaladevuni e Alouini [9]. Em [3], a expressão para a probabilidade de erro de bit de 16-QAM e 64-QAM envolve o cálculo de uma integral definida, cujo integrando é o produto da função- $Q$ e uma função exponencial. Em [4], os autores usam o fato de que um sinal recebido em um canal com desvanecimento é sujeito a uma distorção multiplicativa (DM) e ao ruído aditivo usual - assim, após a compensação da DM, o sinal na entrada do detetor pode ser visto como um sinal que apresenta um 
único termo de distorção aditiva, que compreende os efeitos do ruído aditivo original, da DM, e do erro na compensação da DM. Em [4], a função densidade de probabilidade desse termo de distorção aditiva é obtida e usada para determinar a probabilidade de erro de esquemas de modulação. Uma expressão fechada para a BEP do esquema 16-QAM é apresentada em [4]. Baseando-se em [8], Vitthaladevuni e Alouini apresentaram em [9] expressões genéricas para a BEP de constelações hierárquicas.

Em [10], o método de Craig [11] é estendido para determinar a probabilidade de erro de símbolo de esquemas $M$-ários bidimensionais em canais com desvanecimento Dong et al. determinam em [10] a probabilidade de erro de símbolo média, exata, para a constelação 16-QAM em estrela em canais com desvanecimento.

Em um artigo recente [12], os autores mostraram que o canal com desvanecimento Rayleigh pode ser visto como um canal sujeito a ruído aditivo. Esse ruído é modelado como a razão entre uma variável aleatória (v.a.) gaussiana e uma v.a. Rayleigh. A função cumulativa de probabilidade (FCP) unidimensional desse ruído aditivo foi obtida em [12]. No presente trabalho, é apresentado um método [13], baseado nessa FCP, para obter expressões fechadas para a BEP de esquemas de modulação em canais com desvanecimento Rayleigh. Em particular, o método proposto é usado para obter expressões exatas para a BEP das constelações 16-QAM, 64-QAM e 256-QAM sujeitas ao desvanecimento Rayleigh.

\section{PRELIMINARES}

Considere o sistema de comunicações sem fio ilustrado na Figura 1.
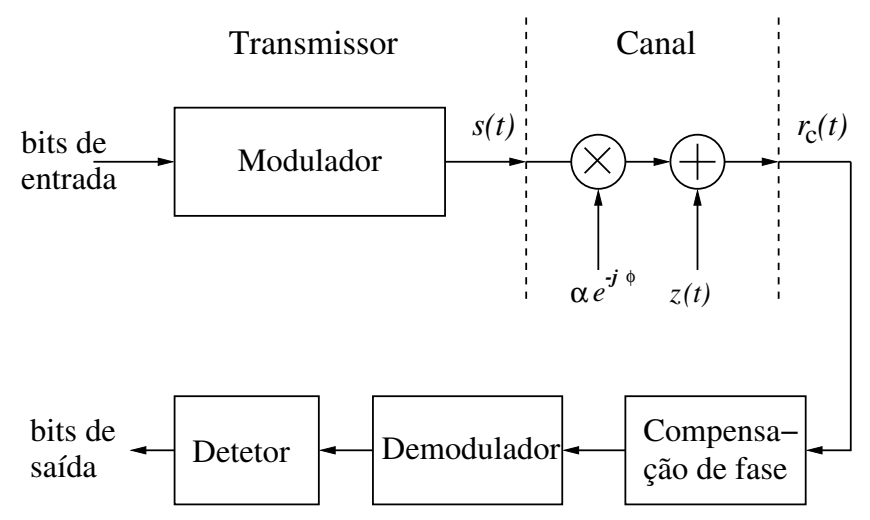

Receptor

Figura 1. Diagrama de blocos de um sistema de comunicações sem fio. O sinal transmitido é afetado pelo desvanecimento e pelo ruído aditivo.

Admitindo que o canal de comunicações é afetado por um desvanecimento lento não-seletivo em freqüência, o sinal recebido $\boldsymbol{r}_{c}(t)$ pode ser expresso como

$$
\boldsymbol{r}_{c}(t)=\alpha e^{-j \phi} \boldsymbol{s}(t)+\boldsymbol{z}(t), \quad 0 \leq t \leq T,
$$

em que $\boldsymbol{s}(t)$ representa o sinal transmitido, $\alpha$ denota a amplitude do desvanecimento, $\phi$ representa o desvio de fase devido ao canal, $\boldsymbol{z}(t)$ denota o ruído aditivo gaussiano branco (AWGN) e $T$ corresponde ao intervalo de sinalização. Usando a representação em passa-baixas, $\boldsymbol{r}_{c}(t)$, $\boldsymbol{s}(t)$ e $\boldsymbol{z}(t)$ são processos estocásticos complexos. Além disso, a condição de desvanecimento lento implica que o parâmetro multiplicativo pode ser considerado constante pelo menos durante um intervalo de sinalização.

A amplitude do desvanecimento $\alpha$ é modelada por uma variável aleatória (v.a.) do tipo Rayleigh, cuja função densidade de probabilidade (fdp) é dada por

$$
p_{\alpha}(\alpha)=2 \alpha e^{-\alpha^{2}} u(\alpha)
$$

em que $u(\cdot)$ representa a função degrau unitário. O ruído aditivo $\boldsymbol{z}(t)$ é um processo estocástico complexo com média nula e densidade espectral de potência $N_{0} / 2$ por dimensão. Considere, sem perda da generalidade, o desvanecimento normalizado, isto é, $E\left[\alpha^{2}\right]=1$, em que $E[\cdot]$ é o operador valor esperado.

Supondo que o desvanecimento que afeta o canal é suficientemente lento, o desvio de fase $\phi$ pode ser estimado sem erro a partir do sinal recebido. Neste caso, o receptor pode compensar o desvio de fase provocado pelo canal (multiplicação de $\boldsymbol{r}_{c}(t)$ por $e^{j \phi}$ ). Então, o sinal recebido resultante $\boldsymbol{r}(t)$ pode ser expresso como

$$
\begin{aligned}
\boldsymbol{r}(t) & =\boldsymbol{r}_{c}(t) \cdot e^{j \phi}=\alpha \boldsymbol{s}(t)+\boldsymbol{z}(t) \cdot e^{j \phi} \\
& =\alpha \boldsymbol{s}(t)+\boldsymbol{n}(t)
\end{aligned}
$$

É importante notar que o ruído aditivo $\boldsymbol{n}(t)=\boldsymbol{z}(t) \cdot e^{j \phi}$ é também um processo estocástico complexo com média nula e densidade espectral de potência $N_{0} / 2$ em suas partes real e imaginária. Isto ocorre porque a distribuição de probabilidade bidimensional $p_{N}(n)$ não é afetada pela compensação de fase, visto que esta distribuição é esfericamente simétrica [14, pp. 247].

A partir de agora, será adotada a notação da representação vetorial dos sinais. Nesta representação, cada sinal $\boldsymbol{s}(t)$ é completamente determinado por um vetor $s$ cujas componentes representam a decomposição do sinal em um conjunto de funções ortonormais escolhido de forma conveniente [14].

O critério de máxima probabilidade a posteriori [7] estabelece que o detetor ótimo, a partir da observação de $\boldsymbol{r}$, seleciona $\tilde{s}=s_{k}$ como o símbolo demodulado quando a função de decisão

$$
P\left(\boldsymbol{s}_{i}\right) p_{r}\left(\boldsymbol{r} \mid \boldsymbol{s}=\boldsymbol{s}_{i}\right), \quad i=0,1,2, \ldots M-1,
$$

é máxima para $i=k$.

Baseando-se no critério de máxima probabilidade a posteriori e considerando que os símbolos da constelação são equiprováveis, o receptor pode utilizar duas estratégias diferentes para determinar o símbolo transmitido mais provável a partir da observação ruidosa $\boldsymbol{r}$ :

- Estratégia I (EI): Amplificar o sinal recebido para normalizar o ganho do canal, de modo que as regiões de decisão no receptor correspondam às regiões de decisão da constelação dos sinais transmitidos, ou seja, deve-se comparar $\boldsymbol{r}$ com todos os símbolos da constelação (multiplicados por $\alpha$ ) e escolher como símbolo demodulado 
o mais próximo a $\boldsymbol{r}$, isto é, o símbolo que minimiza a métrica $\left|\boldsymbol{r}-\alpha \boldsymbol{s}_{i}\right|$;

- Estratégia II (EII): Usar o ganho do canal para amplificar a constelação dos sinais e determinar as regiões de decisão do receptor, ou seja, deve-se comparar $\boldsymbol{r} / \alpha$ com todos os símbolos da constelação e escolher como símbolo demodulado o mais próximo a $\boldsymbol{r} / \alpha$, isto é, o símbolo que minimiza a métrica $\left|\boldsymbol{r} / \alpha-\boldsymbol{s}_{i}\right|$.

Em se tratando de QPSK, mostrou-se em [12] que ambas estratégias (EI e EII) apresentam o mesmo desempenho em termos de probabilidade de erro de bit. No que diz respeito à complexidade, mostrou-se que a estratégia EII constitui uma alternativa mais interessante, visto que requer um número de operações inferior ao requerido pela estratégia EI. Em se tratando da estratégia EII, o canal com desvanecimento Rayleigh, após compensação de desvanecimento (divisão de $\boldsymbol{r}$ por $\alpha$ ), funciona como um canal sujeito a ruído aditivo - esse ruído é modelado como a razão entre uma variável aleatória (v.a.) de Gauss e uma v.a. com distribuição Rayleigh. No presente artigo, a função cumulativa unidimensional de probabilidade da v.a. referente a esse ruído aditivo (que corresponde a $\boldsymbol{n} / \alpha$ ), obtida em [12], é usada na determinação de expressões exatas para a probabilidade de erro de bit dos esquemas de modulação 16-QAM, 64-QAM e 256-QAM em um canal com desvanecimeto Rayleigh. São apresentados resultados de simulação que corroboram as expressões analíticas obtidas no presente trabalho.

$\mathrm{O}$ restante do artigo encontra-se organizado de acordo com as seções a seguir. A Seção 3 aborda o esquema de demodulação em que se utiliza a estratégia EII e, para manter o artigo auto-contido, o obtenção da FCP correspondente à v.a. que modela o ruído aditivo $n / \alpha$ é revisitada. Nas Seções 4,5 e 6 são obtidas expressões para probabilidade de erro de bit (BEP), considerando um canal com desvanecimento Rayleigh, dos esquemas de modulação 16-QAM, 64QAM e 256-QAM, respectivamente. Na Seção 7 são apresentados os comentários finais do trabalho.

\section{DEMODULAÇÃO EM UM CANAL COM DESVANECIMENTO RAYLEIGH}

Nesta seção é abordada a demodulação considerando a estratégia EII, cuja regra de decisão é

$$
\tilde{\boldsymbol{s}}=\arg \min _{\boldsymbol{s}_{i}}\left(\left|\frac{\boldsymbol{r}}{\alpha}-\boldsymbol{s}_{i}\right|\right) \quad i=0,1,2, \cdots, M-1 .
$$

$\mathrm{Na}$ expressão anterior, a função $\arg \min (\cdot)$ retorna o símbolo $s_{i}$ que miniza o seu argumento.

Neste esquema, após a compensação do desvanecimento (divisão de $\boldsymbol{r}$ por $\alpha$ ), o canal pode ser interpretado como um canal sujeito a ruído aditivo porque

$$
\begin{aligned}
\tilde{\boldsymbol{s}} & =\arg \min _{\boldsymbol{s}_{i}}\left(\left|\frac{\alpha \boldsymbol{s}+\boldsymbol{n}}{\alpha}-\boldsymbol{s}_{i}\right|\right) \\
& =\arg \min _{\boldsymbol{s}_{i}}\left(\left|\boldsymbol{s}+\frac{\boldsymbol{n}}{\alpha}-\boldsymbol{s}_{i}\right|\right) \\
& =\arg \min _{\boldsymbol{s}_{i}}\left(\left|\boldsymbol{s}+\boldsymbol{m}-\boldsymbol{s}_{i}\right|\right),
\end{aligned}
$$

em que $\boldsymbol{m}=\boldsymbol{n} / \alpha$ é o ruído aditivo, o qual é modelado como uma v.a. correspondente ao quociente entre entre uma v.a gaussiana e uma v.a. com distribuição Rayleigh.

\subsection{A FUNÇÃO DENSIDADE DE PROBABI- LIDADE E A FUNÇÃO CUMULATIVA DE PROBABILIDADE DO RUÍDO $\boldsymbol{m}$}

Esta seção apresenta a função densidade de probabilidade (fdp) e a função cumulativa de probabilidade (FCP) da variável aleatória (v.a.) obtida pela razão entre uma v.a. gaussiana e uma v.a. Rayleigh. Essa v.a. pode ser expressa como $\mathcal{M}=N / \alpha$, em que $N$ denota uma v.a. aleatória gaussiana com média zero e variância $N_{0} / 2$, ou seja,

$$
p_{N}(n)=\frac{1}{\sqrt{\pi N_{0}}} e^{-n^{2} / N_{0}}
$$

Por sua vez, $\alpha$ é uma v.a. com distribuição de Rayleigh dada pela Equação (2).

Sob essas condições, a fdp de $\mathcal{M}$ é dada por [15]

$$
p_{\mathcal{M}}(m)=\int_{-\infty}^{\infty}|\alpha| p_{N \alpha}(m \alpha, \alpha) d \alpha
$$

em que $p_{N \alpha}(n, \alpha)$ é a função densidade de probabilidade conjunta de $N$ e $\alpha$, dada por

$$
p_{N \alpha}(n, \alpha)=\frac{2}{\sqrt{\pi N_{0}}} \alpha e^{-\left(\alpha^{2}+n^{2} / N_{0}\right)} u(\alpha) .
$$

Observe que, na expressão acima, as variáveis $N$ e $\alpha$ foram cosideradas independentes.

Desta forma, a fdp de $\mathcal{M}$ é dada por

$$
\begin{aligned}
p_{\mathcal{M}}(m) & =\int_{0}^{\infty} \alpha \frac{2}{\sqrt{\pi N_{0}}} \alpha e^{-\left(\alpha^{2}+m^{2} \alpha^{2} / N_{0}\right)} d \alpha \\
& =\frac{2}{\sqrt{\pi N_{0}}} \int_{0}^{\infty} \alpha^{2} e^{-\alpha^{2}\left(1+m^{2} / N_{0}\right)} d \alpha .
\end{aligned}
$$

Usando o fato de que [16, pp. 1030]

$$
\int_{0}^{\infty} x^{2} e^{-x^{2}} d x=\frac{\sqrt{\pi}}{4}
$$

pode-se mostrar que

$$
\int_{0}^{\infty} x^{2} e^{-\rho x^{2}} d x=\frac{1}{\rho^{3 / 2}} \cdot \frac{\sqrt{\pi}}{4} .
$$

Então, a Equação (9) pode ser escrita como

$$
\begin{aligned}
p_{\mathcal{M}}(m) & =\frac{2}{\sqrt{\pi N_{0}}} \cdot \frac{1}{\left(m^{2} / N_{0}+1\right)^{3 / 2}} \cdot \frac{\sqrt{\pi}}{4} \\
& =\frac{1}{2} \cdot \frac{N_{0}}{\left(m^{2}+N_{0}\right)^{3 / 2}} .
\end{aligned}
$$

Finalmente, a FCP de $\mathcal{M}, P_{\mathcal{M}}(m)$, é obtida integrando a expressão anterior, usando [17, Equação 2.264]. Assim, a FCP de $\mathcal{M}$ é dada por

$$
P_{\mathcal{M}}(m)=\int_{-\infty}^{m} p_{\mathcal{M}}(x) d x=\frac{1}{2}\left(\frac{m}{\sqrt{m^{2}+N_{0}}}+1\right) .
$$


O método apresentado no presente trabalho para obter expressões fechadas para a probabilidade de erro de bit de esquemas de modulação sujeitos ao desvanecimento Rayleigh consiste em usar a FCP de $\mathcal{M}$.

As próximas seções mostram como essa FCP é usada para obter expressões exatas para a probabilidade de erro de bit dos sistemas 16-QAM, 64-QAM e 256-QAM em um canal com desvanecimento Rayleigh.

\section{BEP PARA O SISTEMA 16-QAM EM UM CANAL COM DESVANCIMENTO RAYLEIGH}

No sistema 16-QAM, a informação a ser transmitida é mapeada em símbolos com 4 bits e cada símbolo é usado para definir uma única amplitude e uma única fase da portadora. A duração de cada símbolo determina a largura de faixa do sinal 16-QAM. A Figura 2 ilustra uma constelação com 16 níveis em que cada ponto corresponde a um símbolo 16-QAM.

Os símbolos da constelação 16-QAM podem ser vistos como dois sinais PAM (cada um com quatro níveis) que são transmitidos em fase e em quadratura na mesma frequiência $[3,5,7,18]$. Cada uma das portadoras PAM é transmitida com uma amplitude do conjunto $(-3 d,-d, d$, $3 d$ ), sendo $2 d$ a distância entre os símbolos de acordo com a Figura 2. Um código Gray de 2 bits é usado para associar cada par de bits a uma amplitude da portadora PAM. Os bits $11,10,00$ e 01 são associados aos níveis $-3 d,-d, d$ e $3 d$, respectivamente. Os símbolos 16-QAM são obtidos a partir do entrelaçamento dos bits em quadratura e em fase. Um símbolo 16-QAM apresenta a forma $\left(i_{1} q_{1} i_{2} q_{2}\right)$, em que $i_{1} i_{2}$ representa os bits correspondentes à componente em fase e $q_{1} q_{2}$ representa os bits correspondentes à componente em quadratura [5].

A demodulação QAM é realizada demodulando independentemente as componentes $Q$ e $I$ usando as respectivas fronteiras de decisão ilustradas na Figura 2 [3,5], a qual também mostra as regiões em que os bits são um, e.g., $q_{2}=1$ para $Q<-2 d$ ou $Q>2 d$. A Figura 2 também revela que em metade do tempo os bits $i_{1}$ e $q_{1}$ têm uma distância de proteção de ruído $d$ de suas respectivas fronteiras de decisão, enquanto que metade do tempo esta distância é $3 d$. Os bits $i_{2}$ e $q_{2}$ estão sempre a uma distância $d$ das suas respectivas fronteiras de decisão, estando, portanto, mais propensos a erros quando comparados a $i_{1}$ e $q_{1}$. Devido a essa propriedade, o esquema 16-QAM pode ser considerado como a combinação de dois subcanais com diferentes integridades, denominados subcanais classe 1 e classe 2 ( $\mathrm{C} 1$ e $\mathrm{C} 2$ ). O processo de demodulação referente ao subcanal $\mathrm{C} 1$ é descrito analiticamente como segue $[3,5]$ :

$$
\begin{aligned}
& \text { se } I, Q \geq 0, \quad \text { então } i_{1}, q_{1}=0 \text {, } \\
& \text { se } I, Q<0, \text { então } i_{1}, q_{1}=1 \text {. }
\end{aligned}
$$

Levando em consideração as fronteiras de decisão para o terceiro e o quarto bits, $i_{2}$ e $q_{2}$, conforme mostrado na

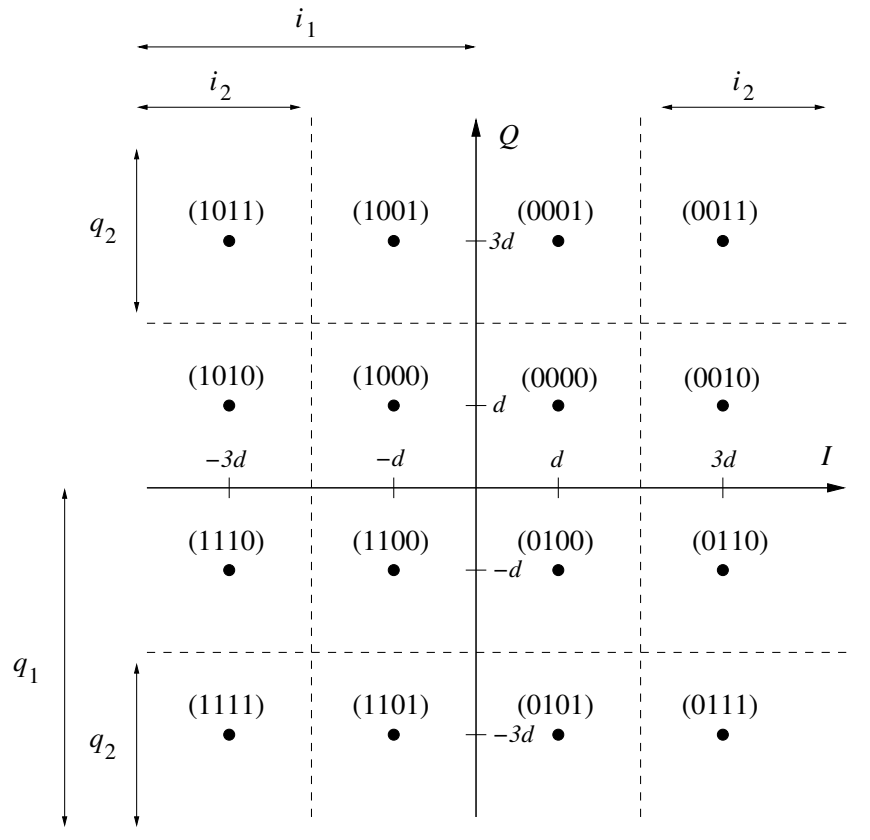

Figura 2. Constelação 16-QAM. As palavras-binárias $i_{1} q_{1} i_{2} q_{2}$ para cada símbolo são apresentadas. As regiões em que cada bit $\left(i_{1}, i_{2}, q_{1}\right.$ e $\left.q_{2}\right)$ é igual a um também estão indicadas.

Figura 2, tem-se:

$$
\begin{aligned}
& \text { se } I, Q \geq 2 d, \quad \text { então } i_{2}, q_{2}=1, \\
& \text { se } \quad-2 d \leq I, Q<2 d, \quad \text { então } i_{2}, q_{2}=0, \\
& \text { se } I, Q<-2 d, \quad \text { então } \quad i_{2}, q_{2}=1 .
\end{aligned}
$$

A probabilidade de erro de bit pode ser calculada considerando a transmissão independente das componentes em fase e em quadratura $(I$ e $Q)$ apresentadas na Figura 3. Logo, a probabilidade de erro de bit do esquema 16-QAM pode ser expressa como

$$
P_{16-\mathrm{QAM}}=\frac{P(E \mid 11)+P(E \mid 10)+P(E \mid 00)+P(E \mid 01)}{4},
$$

em que $P\left(E \mid b_{1} b_{2}\right)$ representa a probabilidade de erro de bit dado que os bits $b_{1} b_{2}$ foram transmitidos. A partir da simetria apresentada na Figura 3, conclui-se que $P(E \mid 11)=P(E \mid 01)$ e $P(E \mid 10)=P(E \mid 00)$. Desta forma, a Equação (14) ser reduz a

$$
P_{16-\mathrm{QAM}}=\frac{P(E \mid 11)+P(E \mid 10)}{2} .
$$

\begin{tabular}{ccccc}
$-3 d$ & $-d$ & $d$ & $3 d$ & I ou $Q$ \\
\hline & $\mid$ & $\mid$ & $\mid$
\end{tabular}

Figura 3. Componentes da constelação 16-QAM.

A probabilidade de erro de bit $P(E \mid 11)$ pode ser calculada como

$$
P(E \mid 11)=\frac{1}{2} P(10 \mid 11)+\frac{2}{2} P(00 \mid 11)+\frac{1}{2} P(01 \mid 11),
$$


em que $P\left(c_{1} c_{2} \mid b_{1} b_{2}\right)$ representa a probabilidade de recepção dos bits $c_{1} c_{2}$ dado que os bits $b_{1} b_{2}$ foram transmitidos.

A partir da Figura 3 pode-se escrever $P(E \mid 11)$ em termos de probabilidades relacionadas à variável $m$, ou seja,

$$
\begin{aligned}
P(E \mid 11)= & \frac{1}{2} P(d \leq m<3 d)+\frac{2}{2} P(3 d \leq m<5 d) \\
& +\frac{1}{2} P(m \geq 5 d) .
\end{aligned}
$$

Usando a Equação (13), tem-se:

$$
\begin{aligned}
P(E \mid 11)= & \frac{1}{2}\left[\left.\frac{1}{2}\left(\frac{m}{\sqrt{m^{2}+N_{0}}}+1\right)\right|_{d} ^{3 d}\right] \\
& +\frac{2}{2}\left[\left.\frac{1}{2}\left(\frac{m}{\sqrt{m^{2}+N_{0}}}+1\right)\right|_{3 d} ^{5 d}\right] \\
& +\frac{1}{2}\left[\left.\frac{1}{2}\left(\frac{m}{\sqrt{m^{2}+N_{0}}}+1\right)\right|_{5 d} ^{+\infty}\right] \\
= & \frac{1}{4}\left[1-\frac{d}{\sqrt{d^{2}+N_{0}}}-\frac{3 d}{\sqrt{9 d^{2}+N_{0}}}\right. \\
& \left.+\frac{5 d}{\sqrt{25 d^{2}+N_{0}}}\right] .
\end{aligned}
$$

De forma análoga,

$$
P(E \mid 10)=\frac{3}{4}-\frac{1}{2} \frac{d}{\sqrt{d^{2}+N_{0}}}-\frac{1}{4} \frac{3 d}{\sqrt{9 d^{2}+N_{0}}} .
$$

Substituindo as Equações (18) e (19) na Equação (15), a probabilidade de erro de bit para a constelação 16-QAM pode ser expressa como

$$
\begin{aligned}
P_{16-\mathrm{QAM}}= & \frac{1}{2}-\frac{3}{8} \frac{d}{\sqrt{d^{2}+N_{0}}}-\frac{1}{4} \frac{3 d}{\sqrt{9 d^{2}+N_{0}}} \\
& +\frac{1}{8} \frac{5 d}{\sqrt{25 d^{2}+N_{0}}} .
\end{aligned}
$$

A partir da Figura 2, observa-se que a energia média por símbolo da constelação, $E_{S}$, é dada por

$$
E_{S}=10 d^{2} \text {. }
$$

Como no esquema 16-QAM cada conjunto de 4 bits é mapeado em um símbolo da constelação, a energia média por bit $E_{b}$ é dada por

$$
E_{b}=2,5 d^{2} .
$$

Substituindo a Equação (22) na Equação (20) e definindo a seguinte função auxiliar

$$
\Theta\left(E_{b} / N_{0}, a\right) \triangleq \sqrt{\frac{a \cdot E_{b} / N_{0}}{a \cdot E_{b} / N_{0}+1}},
$$

obtém-se que a expressão final para a BEP do sistema de modulação 16-QAM em função da relação sinal-ruído média,
$E_{b} / N_{0}$, é dada por

$$
\begin{aligned}
P_{16-\mathrm{QAM}}=\frac{1}{2} & -\frac{3}{8} \Theta\left(E_{b} / N_{0}, 0,4\right) \\
& -\frac{1}{4} \Theta\left(E_{b} / N_{0}, 3,6\right) \\
& +\frac{1}{8} \Theta\left(E_{b} / N_{0}, 10\right) .
\end{aligned}
$$

Convém ressaltar que a abordagem apresentada neste trabalho para a obtenção da BEP para 16-QAM difere da abordagem apresentada em [4] - no artigo de Shayesteh e Aghamohammadi não é considerada a demodulação independente das componentes em fase e quadratura dos símbolos transmitidos. No presente trabalho, diferentemente de [4], utiliza-se a fdp unidimensional do novo ${ }^{1}$ ruído aditivo. Isso é possível devido ao mapeamento binário utilizado, que faz com que a componente em quadratura do ruído não tenha influência na probabilidade de erro dos bits da componente em fase, e vice-versa ${ }^{2}$. A expressão obtida em [4] é dada por

$$
\begin{aligned}
P_{16-\text { QAm }, \text { Shay }} & =\frac{1}{2}-\frac{1}{4} \Theta\left(E_{b} / N_{0}, 0,4\right) \\
& \cdot\left\{1+\frac{1}{\pi} \tan ^{-1}\left(3 \Theta\left(E_{b} / N_{0}, 0,4\right)\right)\right\} \\
& -\frac{1}{4} \Theta\left(E_{b} / N_{0}, 3,6\right) \\
& \cdot\left\{1+\frac{1}{\pi} \tan ^{-1}\left(\frac{1}{3} \Theta\left(E_{b} / N_{0}, 3,6\right)\right)\right\} \\
& +\frac{1}{8} \Theta\left(E_{b} / N_{0}, 10\right) .
\end{aligned}
$$

Observa-se que a expressão obtida no presente trabalho é mais compacta que a apresentada em [4]. Além disso, a Expressão (24) não contém funções trancendentais (como $\tan ^{-1}$ ), que ocorrem na Expressão (25). Convém salientar ainda que as Expressões (24) e (25) são assintoticamente idênticas para $E_{b} / N_{0} \gg 1$.

A Figura 4 apresenta o gráfico da probabilidade de erro de bit do sistema 16-QAM em função da relação sinal-ruído, $E_{b} / N_{0}$, em dB. Observa-se que os resultados de simulação corroboram tanto a Expressão (24) quanto a expressão obtida por Shayesteh e Aghamohammadi. Nota-se também, a partir da Expressão (24), que a probabilidade de erro de bit não apresenta um patamar de erro irredutível. Isto advém do fato de que

$$
\lim _{E_{b} / N_{0} \rightarrow \infty} P_{16-\mathrm{QAM}}=0
$$

pois

$$
\lim _{E_{b} / N_{0} \rightarrow \infty} \Theta\left(E_{b} / N_{0}, a\right)=1 .
$$

Na Figura 4 também foram incluídos resultados considerando o canal AWGN [8]. Como era esperado, observa-se que o desvanecimento provoca um aumento na probabilidade de erro de bit.

\footnotetext{
${ }^{1}$ Resultante da divisão do ruído gaussiano $(n)$ pelo desvanecimento $(\alpha)$.

${ }^{2}$ Cumpre mencionar que as componentes em quadratura desse novo ruído aditivo são dependentes.
} 


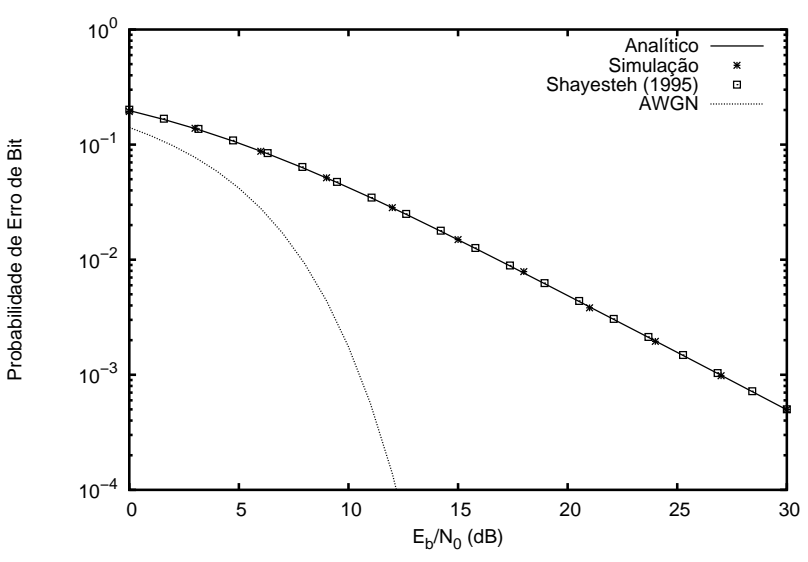

Figura 4. Probabilidade de erro de bit para a constelação 16-QAM em um canal com desvanecimento Rayleigh em função da relação sinal-ruído $\left(E_{b} / N_{0}\right)$. A curva considerando o canal AWGN também foi incluída para fins de comparação.

\section{BEP PARA O SISTEMA 64-QAM EM UM CANAL COM DESVANCIMENTO RAYLEIGH}

A Figura 5 mostra a constelação, as fronteiras de decisão e o mapeamento em bits para o esquema 64-QAM. Observase que o primeiro, o terceiro e o quinto bits correspondem à cadeia de bits em fase, enquanto que os demais bits correspondem à cadeia de bits em quadratura. Um código Gray de 3 bits é usado nessas componentes individuais $I$ e $Q-$ as palavras 011, 010, 000, 001, 101, 100, 110 e 111 são atribuídas aos níveis $7 d, 5 d, 3 d, d,-d,-3 d,-5 d$ e $-7 d$, respectivamente, conforme mostra a Figura 6 . Os bits em fase e quadratura são entrelaçados, gerando um símbolo QAM de 6 bits, representado por $i_{1} q_{1} i_{2} q_{2} i_{3} q_{3}$. A Figura 5 também mostra as regiões em que os bits são um, e.g., $q_{2}=1$ para $Q<-4 d$ ou $Q>4 d$.

A demodulação é realizada usando as fronteiras de decisão mostradas na Figura 6 e as seguintes equações [3,5]:

$$
\begin{array}{lll}
\text { se } \quad I, Q \geq 0, & \text { então } & i_{1}, q_{1}=0, \\
\text { se } \quad I, Q<0, & \text { então } & i_{1}, q_{1}=1,
\end{array}
$$

para os bits mais significativos,

$$
\begin{aligned}
& \text { se } \quad I, Q \geq 4 d, \quad \text { então } i_{2}, q_{2}=1, \\
& \text { se } \quad-4 d \leq I, Q<4 d, \quad \text { então } i_{2}, q_{2}=0, \\
& \text { se } \quad-4 d>I, Q, \quad \text { então } i_{2}, q_{2}=1,
\end{aligned}
$$

para os próximos bits mais significativos, e, finalmente,

$$
\begin{aligned}
& \text { se } \quad I, Q \geq 6 d, \quad \text { então } i_{3}, q_{3}=1, \\
& \text { se } \quad 2 d \leq I, Q<6 d, \quad \text { então } i_{3}, q_{3}=0, \\
& \text { se } \quad-2 d \leq I, Q<2 d, \quad \text { então } i_{3}, q_{3}=1, \\
& \text { se } \quad-6 d \leq I, Q<-2 d, \quad \text { então } i_{3}, q_{3}=0, \\
& \text { se } \quad-6 d>I, Q, \quad \text { então } i_{3}, q_{3}=1,
\end{aligned}
$$

A probabilidade de erro de bit do esquema 64-QAM pode ser obtida considerando transmissão independente das componentes em fase $(I)$ e quadratura $(Q)$, mostradas na Figura 6. A BEP, portanto, pode ser expressa como

$$
\begin{aligned}
P_{64-\mathrm{QAM}}= & \frac{1}{8}[P(E \mid 111)+P(E \mid 110)+P(E \mid 100) \\
& +P(E \mid 101)+P(E \mid 001)+P(E \mid 000) \\
& +P(E \mid 010)+P(E \mid 011)],
\end{aligned}
$$

em que $P\left(E \mid b_{1} b_{2} b_{3}\right)$ denota a probabilidade de erro de bit dado que os bits $b_{1} b_{2} b_{3}$ foram transmitidos. Pela simetria da Figura 6, tem-se que $P(E \mid 111)=P(E \mid 011)$, $P(E \mid 110)=P(E \mid 010), P(E \mid 100)=P(E \mid 000) \mathrm{e}$ $P(E \mid 101)=P(E \mid 001)$. Portanto, (28) reduz-se a

$$
\begin{aligned}
P_{64-\mathrm{QAM}}= & \frac{1}{4}\{P(E \mid 111)+P(E \mid 110) \\
& +P(E \mid 100)+P(E \mid 101)\} .
\end{aligned}
$$

Observando a Figura 6, $P(E \mid 111)$ pode ser escrita em termos de probabilidades relacionadas a $m$, ou seja,

$$
\begin{aligned}
& P(E \mid 111)=\frac{1}{3} P(d \leq m<3 d) \\
& +\frac{2}{3} P(3 d \leq m<5 d)+\frac{1}{3} P(5 d \leq m<7 d) \\
& +\frac{2}{3} P(7 d \leq m<9 d)+\frac{3}{3} P(9 d \leq m<11 d) \\
& +\frac{2}{3} P(11 d \leq m<13 d)+\frac{1}{3} P(m \geq 13 d) .
\end{aligned}
$$

Usando (13), a equação anterior pode ser dada por

$$
\begin{aligned}
P(E \mid 111)= & \left.\left.\frac{1}{3}\left[\frac{m}{\sqrt{m^{2}+N_{0}}}+1\right)\right|_{d} ^{3 d}\right] \\
+ & \frac{2}{3}\left[\left.\frac{1}{2}\left(\frac{m}{\sqrt{m^{2}+N_{0}}}+1\right)\right|_{3 d} ^{5 d}\right] \\
+ & \frac{1}{3}\left[\left.\frac{1}{2}\left(\frac{m}{\sqrt{m^{2}+N_{0}}}+1\right)\right|_{5 d} ^{7 d}\right] \\
+ & \frac{2}{3}\left[\left.\frac{1}{2}\left(\frac{m}{\sqrt{m^{2}+N_{0}}}+1\right)\right|_{7 d} ^{9 d}\right] \\
+ & \frac{3}{3}\left[\left.\frac{1}{2}\left(\frac{m}{\sqrt{m^{2}+N_{0}}}+1\right)\right|_{9 d} ^{11 d}\right] \\
+ & \frac{2}{3}\left[\left.\frac{1}{2}\left(\frac{m}{\sqrt{m^{2}+N_{0}}}+1\right)\right|_{11 d} ^{13 d}\right] \\
& +\frac{1}{3}\left[\left.\frac{1}{2}\left(\frac{m}{\sqrt{m^{2}+N_{0}}}+1\right)\right|_{13 d} ^{+\infty}\right] .
\end{aligned}
$$

Seja

$$
f(x)=\frac{1}{2} \cdot \frac{x}{\sqrt{x^{2}+N_{0}}}
$$

para os bits menos significativos. 


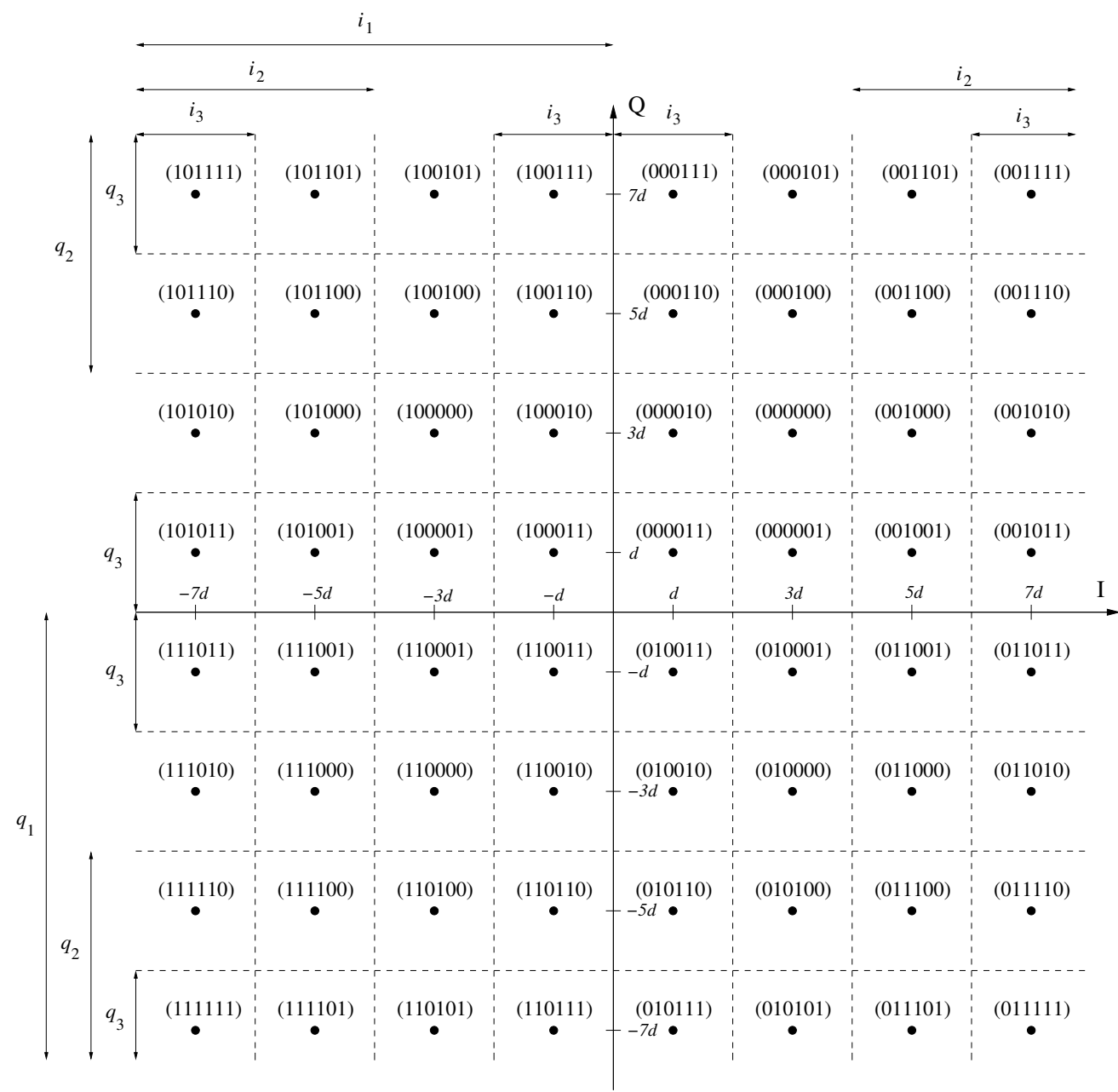

Figura 5. Constelação 64-QAM. As palavras-binárias $i_{1} q_{1} i_{2} q_{2} i_{3} q_{3}$ para cada símbolo são apresentadas. As regiões em que cada bit $\left(i_{1}, i_{2}, i_{3}, q_{1}, q_{2}\right.$ e $\left.q_{3}\right)$ é igual a um também estão indicadas.

\begin{tabular}{cccc|ccccc}
$-7 d$ & $-5 d$ & $-3 d$ & $-d$ & $d$ & $3 d$ & $5 d$ & $7 d$ & I ou $Q$ \\
\hline$(111)$ & $(110)$ & $(100)$ & $(101)$ & $(001)$ & $(000)$ & $(010)$ & $(011)$
\end{tabular}

Figura 6. Componentes da constelação 64-QAM.

Assim, usando (32), a expressão (31) pode ser reescrita como

$$
\begin{aligned}
& P(E \mid 111)=\frac{1}{3}[f(3 d)-f(d)]+\frac{2}{3}[f(5 d)-f(3 d)] \\
& \quad+\frac{1}{3}[f(7 d)-f(5 d)]+\frac{2}{3}[f(9 d)-f(7 d)] \\
& \quad+\frac{3}{3}[f(11 d)-f(9 d)]+\frac{2}{3}[f(13 d)-f(11 d)] \\
& \quad+\frac{1}{3}[1 / 2-f(13 d)] .
\end{aligned}
$$

De forma semelhante,

$$
\begin{aligned}
& P(E \mid 110)=\frac{1}{3}[1 / 2-f(d)]+\frac{1}{3}[f(3 d)-f(d)] \\
& +\frac{2}{3}[f(5 d)-f(3 d)]+\frac{3}{3}[f(7 d)-f(5 d)] \\
& +\frac{2}{3}[f(9 d)-f(7 d)]+\frac{1}{3}[f(11 d)-f(9 d)] \\
& \quad+\frac{2}{3}[1 / 2-f(11 d)],
\end{aligned}
$$

$$
\begin{aligned}
& P(E \mid 100)=\frac{2}{3}[1 / 2-f(3 d)]+\frac{1}{3}[f(3 d)-f(d)] \\
& \quad+\frac{1}{3}[f(3 d)-f(d)]+\frac{2}{3}[f(5 d)-f(3 d)] \\
& \quad+\frac{1}{3}[f(7 d)-f(5 d)]+\frac{2}{3}[f(9 d)-f(7 d)] \\
& \quad+\frac{3}{3}[1 / 2-f(9 d)],
\end{aligned}
$$

e

$$
\begin{aligned}
& P(E \mid 101)=\frac{1}{3}[1 / 2-f(5 d)]+\frac{2}{3}[f(5 d)-f(3 d)] \\
& +\frac{1}{3}[f(3 d)-f(d)]+\frac{1}{3}[f(3 d)-f(d)] \\
& +\frac{2}{3}[f(5 d)-f(3 d)]+\frac{3}{3}[f(7 d)-f(5 d)] \\
& \quad+\frac{2}{3}[1 / 2-f(7 d)] .
\end{aligned}
$$


Substituindo (33)-(36) em (29), tem-se

$$
\begin{aligned}
P_{64-\mathrm{QAM}}= & \frac{1}{4}\left[2-\frac{7}{3} f(d)-\frac{6}{3} f(3 d)+\frac{1}{3} f(5 d)\right. \\
& \left.-\frac{1}{3} f(9 d)+\frac{1}{3} f(13 d)\right]
\end{aligned}
$$

Observando a Figura 5, a energia média por símbolo da constelação, $E_{S}$, é dada por

$$
E_{S}=42 d^{2}
$$

Tendo em vista que cada seqüência de 6 bits é mapeada em um símbolo da constelação, a energia média por bit, $E_{b}$, é dada por

$$
E_{b}=7 d^{2}
$$

Substituindo (39) em (37), e levando em consideração (32), a expressão para a BEP do esquema 64-QAM em função de $E_{b} / N_{0}$ é finalmente dada por

$$
\begin{aligned}
P_{64-\mathrm{QAM}}= & \frac{1}{24}\left[12-7 \Theta\left(E_{b} / N_{0}, 1 / 7\right)\right. \\
& -6 \Theta\left(E_{b} / N_{0}, 9 / 7\right)+\Theta\left(E_{b} / N_{0}, 25 / 7\right) \\
& \left.-\Theta\left(E_{b} / N_{0}, 81 / 7\right)+\Theta\left(E_{b} / N_{0}, 169 / 7\right)\right] .
\end{aligned}
$$

Conforme se pode observar na Figura 7, a Equação (40) é corroborada por resultados de simulação de Monte Carlo.

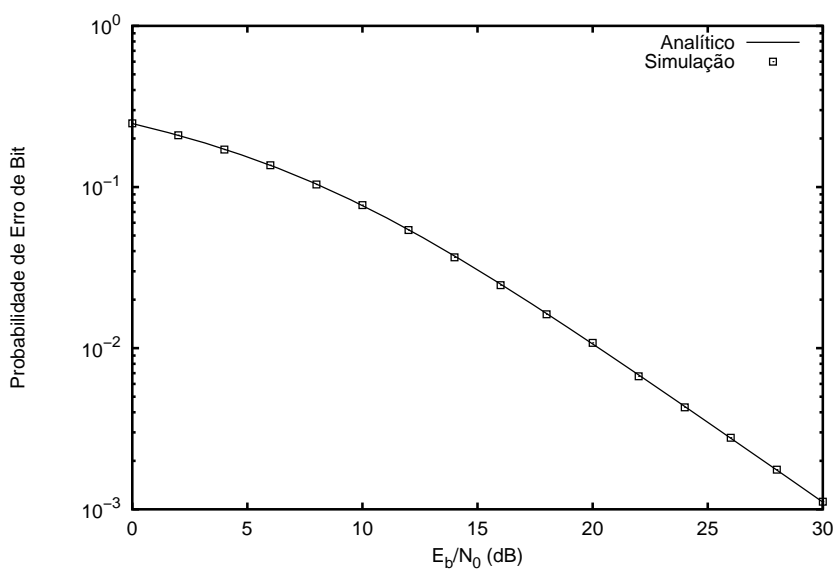

Figura 7. Probabilidade de erro de bit para a constelação 64-QAM em um canal com desvanecimento Rayleigh, em função da relação sinal-ruído $\left(E_{b} / N_{0}\right)$.

\section{BEP PARA O SISTEMA 256-QAM EM UM CANAL COM DESVANCIMENTO RAYLEIGH}

Repetindo o procedimento usado nas duas seções anteriores, pode-se determinar a expressão exata para a probabilidade de erro de bit do esquema 256-QAM em um canal com desvanecimento Rayleigh, que é dada por

$$
\begin{aligned}
& P_{256-\mathrm{QAM}}=\frac{1}{64}\left[32-15 \Theta\left(E_{b} / N_{0}, 4 / 85\right)\right. \\
& -14 \Theta\left(E_{b} / N_{0}, 36 / 85\right)+\Theta\left(E_{b} / N_{0}, 100 / 85\right) \\
& -5 \Theta\left(E_{b} / N_{0}, 324 / 85\right)-4 \Theta\left(E_{b} / N_{0}, 484 / 85\right) \\
& +5 \Theta\left(E_{b} / N_{0}, 676 / 85\right)+4 \Theta\left(E_{b} / N_{0}, 900 / 85\right) \\
& -5 \Theta\left(E_{b} / N_{0}, 1156 / 85\right)-4 \Theta\left(E_{b} / N_{0}, 1444 / 85\right) \\
& +3 \Theta\left(E_{b} / N_{0}, 1764 / 85\right)+2 \Theta\left(E_{b} / N_{0}, 2116 / 85\right) \\
& \left.-\Theta\left(E_{b} / N_{0}, 2500 / 85\right)+\Theta\left(E_{b} / N_{0}, 3364 / 85\right)\right] .
\end{aligned}
$$

Conforme se pode observar na Figura 8, a Equação (41) é corroborada por resultados de simulação de Monte Carlo.

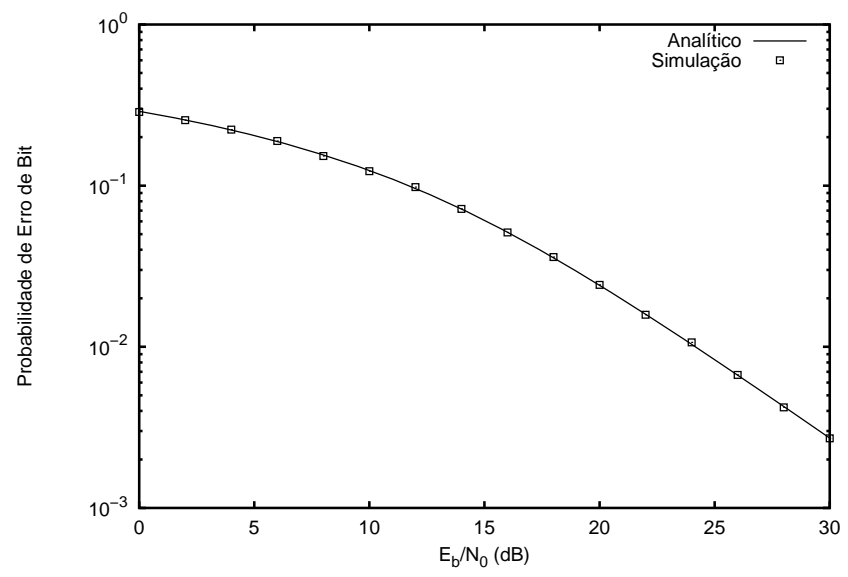

Figura 8. Probabilidade de erro de bit para a constelação 256-QAM em um canal com desvanecimento Rayleigh, em função da relação sinal-ruído $\left(E_{b} / N_{0}\right)$.

Apesar de a FCP em (13) ter sido usada para obter a BEP dos esquemas 16-QAM, 64-QAM e 256-QAM em um canal com desvanecimento Rayleigh, ela pode ser aplicada convenientemente a outros esquemas de modulação, considerando constelações com outros tamanhos.

\section{CONCLUSÃO}

Este artigo apresentou um método conveniente para obter a probabilidade de erro de bit (BEP) de esquemas de modulação em um canal com desvanecimento Rayleigh, o qual é visto como um canal sujeito a ruído aditivo - esse ruído é modelado como a razão entre um processo gaussiano e uma v.a. Rayleigh. O método consiste em usar a função cumulativa de probabilidade (FCP) do processo que modela esse ruído aditivo para obter expressões exatas para a BEP. No presente trabalho, esse método foi usado para obter expressões novas, fechadas, exatas, para a BEP dos esquemas 16-QAM, 64-QAM e 256-QAM sujeitos a desvanecimento Rayleigh. Todas as expressões obtidas para BEP foram corroboradas por resultados de simulação de Monte Carlo. Em trabalhos futuros, o método proposto será usado para obter a BEP de outros esquemas de modulação, considerando constelações com outras dimensões. 


\section{AGRADECIMENTOS}

Os autores gostariam de expressar os seus sinceros agradecimentos aos revisores anônimos, cujos comentários contribuíram sobremaneira para melhoria da qualidade deste artigo. O presente trabalho foi realizado com o apoio do Conselho Nacional de Desenvolvimento Científico e Tecnológico (CNPq).

\section{REFERÊNCIAS}

[1] J. Lu, K. B. Letaief, J. C.-I. Chuang and M. L. Liou. “ $M$-PSK and $M$-QAM BER Computation Using Signal-Space Concepts". IEEE Transactions on Communications, vol. 47, no. 2, pp. 181-184, February 1999.

[2] L.-L. Yang and L. Hanzo. "A Recursive Algorithm for the Error Probability Evaluation of $M$-QAM". IEEE Communications Letters, vol. 4, no. 10, pp. 304-306, October 2000.

[3] P.-M. Fortune, L. Hanzo and R. Steele. "On the Computation of 16-QAM and 64-QAM Performance in Rayleigh-Fading Channels". IEICE Transactions on Communications, vol. E75B, no. 6, pp. 466-475, June 1992.

[4] M. G. Shayesteh and A. Aghamohammadi. "On the Error Probability of Linearly Modulated Signals on Frequency-Flat Ricean, Rayleigh and AWGN Channels". IEEE Transactions on Communications, vol. 43, no. 2/3/4, pp. 1454-1466, February/March/April 1995.

[5] L. Hanzo, R. Steele and P.-M. Fortune. "A Subband Coding, BCH Coding, and 16-QAM System for Mobile Radio Speech Communications". IEEE Transactions on Vehicular Technology, vol. 39, no. 4, pp. 327-339, November 1990.

[6] A. Aghamohammadi and H. Meyr. "On the Error Probability of Linearly Modulated Signals on Rayleigh Frequency-Flat Fading Channels". IEEE Transactions on Communications, vol. 38, no. 11, pp. 1966-1970, November 1990.

[7] J. G. Proakis. Digital Communications. McGraw-Hill, New York, third edition, 1995.

[8] K. Cho and D. Yoon. "On the General BER Expression of One- and Two-Dimensional Amplitude Modulations". IEEE Transactions on Communications, vol. 50, no. 7, pp. 1074 1080, July 2002.

[9] P. K. Vitthaladevuni and M.-S. Alouini. "BER Computation of 4/M-QAM Hierarchical Constellations". IEEE Transactions on Broadcasting, vol. 47, no. 3, pp. 228-239, September 2001.

[10] X. Dong, N. C. Beaulieu and P. H. Wittke. "Error Probabilities of Two-Dimensional $M$-ary Signaling in Fading". IEEE Transactions on Communications, vol. 47, no. 3, pp. 352-355, March 1999.

[11] J. W. Craig. "A New, Simple and Exact Result for Calculating the Probability of Error for Two-Dimensional Signal Constellations". In Proceedings of Military Communications Conference (MILCOM'91), pp. 571-575, 1991.

[12] W. T. A. Lopes and M. S. Alencar. "QPSK Detection Schemes for Rayleigh Fading Channels". In Proceedings of the IEEE International Telecommunications Symposium (ITS'02), Natal, RN, Brazil, September 2002.

[13] W. T. A. Lopes, F. Madeiro and M. S. Alencar. "Um Novo Método para o Cálculo da Probabilidade de Erro de Bit de Esquemas de Modulação Sujeitos ao Desvanecimento Rayleigh". In Anais do XX Simpósio Brasileiro de Telecomunicações (SBT'03), Rio de Janeiro, RJ, Brasil, Outubro 2003.

[14] J. M. Wozencraft and I. M. Jacobs. Principles of Communication Engineering. John Wiley and Sons, 1965.
[15] A. Papoulis. Probability, Random Variables, and Stochastic Processes. McGraw-Hill, New York, third edition, 1991.

[16] A. D. Poularikas, editor. The Transforms and Applications Handbook. IEEE Press, 1996.

[17] I. S. Gradshteyn and I. M. Ryzhik. Table of Integrals, Series, and Products. Academic Press, 1979.

[18] X. Tang, M.-S. Alouini and A. J. Goldsmith. "Effect of Channel Estimation Error on M-QAM BER Performance in Rayleigh Fading”. IEEE Transactions on Communications, vol. 47, no. 12, pp. 1856-1864, December 1999.

Waslon Terllizzie Araújo Lopes nasceu em Petrolina, Pernambuco, em 29 de dezembro de 1974. Recebeu o diploma de Engenheiro Eletricista e o título de Mestre em Engenharia Elétrica pela Universidade Federal da Paraíba em 1998 e 1999, respectivamente. Recebeu o título de Doutor em Engenharia Elétrica pela Universidade Federal de Campina Grande em junho de 2003. Atualmente, Waslon Terllizzie é professor e coordenador do Núcleo de Telecomunicações do Departamento de Engenharia Elétrica da Faculdade ÁREA1, Salvador, BA. É Coordenador executivo do Instituto de Estudos Avançados em Comunicações (Iecom). Suas atividades de pesquisa concentram-se em quantização vetorial robusta, sistemas de comunicações sem fio e teoria das comunicações, áreas em que tem mais de 40 trabalhos publicados. É um dos autores do livro Communications, Information and Network Security, pela Kluwer Academic Publishers. Waslon Terllizzie é membro da Sociedade Brasileira de Telecomunicações (SBrT) desde 1997 e membro da Sociedade Brasileira de Redes Neurais (SBRN).

Francisco Madeiro Bernardino Junior nasceu em Fortaleza, Ceará, em 27 de março de 1972. Recebeu o diploma de Engenheiro Eletricista e os títulos de Mestre e Doutor em Engenharia Elétrica pela Universidade Federal da Paraíba (UFPB) em 1995, 1998 e 2001, respectivamente. Suas atividades de pesquisa concentramse em processamento de sinais e sistemas inteligentes. Francisco Madeiro é professor do Departamento de Estatística e Informática e do Mestrado em Ciências da Linguagem da Universidade Católica de Pernambuco (UNICAP). Foi professor homenageado da turma de concluintes de Ciência da Computação, período 2003.2, da UNICAP. É sócio fundador do Instituto de Estudos Avançados em Comunicações (Iecom), no qual exerce a função de Diretor de Engenharia. É membro da Sociedade Brasileira de Telecomunicações (SBrT), da Sociedade Brasileira de Redes Neurais (SBRN) e da Sociedade Brasileira de Computação (SBC).

Marcelo Sampaio de Alencar nasceu em Serrita, Pernambuco, em 1957. Formou-se em Engenharia Elétrica pela Universidade Federal de Pernambuco, em 1980, recebeu o título de mestre em Engenharia Elétrica pela Universidade Federal da Paraíba, em 1988 e o de Ph.D. em Engenharia Elétrica pela University of Waterloo, Canada, em 1993. Durante o período de 1982 a 1984 trabalhou na Faculdade de Engenharia da Universidade para o Desenvolvimento do Estado de Santa Catarina, onde foi Membro Titular do Conselho Universitário e Vice-Presidente da Associação dos Professores. Entre 1984 e 2001, trabalhou no Departamento de Engenharia Elétrica da Universidade Federal da Paraíba (UFPB). Atualmente trabalha no Departamento de Engenharia Elétrica da Universidade Federal de Campina Grande (UFCG), onde exerce o cargo de Professor Titular. Exerceu os cargos de Vice-Coordenador do Curso de Engenharia Elétrica, Assessor de Extensão do Centro de Ciências e Tecnologia, Presidente da Comissão de Extensão do Centro de Ciências e Tecnologia da UFPB e foi Membro nato do Comitê Assessor de Extensão da Universidade Federal da Paraíba. Atualmente exerce a Coordenação do Grupo de Comunicações do Departamento, com cadastro no CNPq. Trabalhou como consultor 
da Embratel, da Telern, da Telebras, da Chesf, da TIM, da Siemens, da Contol, do CNPq e da Finep em diversos projetos, além de ser Coordenador dos convênios que a UFCG mantém com a Embratel, Telern, Chesf e Atecel. Em 1989, trabalhou na Divisão Regional de Engenharia da Embratel, em Recife. Foi Diretor da Sociedade Brasileira de Telecomunicações (SBrT), membro do Conselho Deliberativo e. atualmente, é Vice-Presidente para Relações Externas. Membro da Sociedade Brasileira de Microondas (SBMO), da Sociedade Brasileira de Engenharia Biomédica (SBEB), da Associação Brasileira de Jornalismo Científico (ABJC) e do Instituto dos Engenheiros Eletricistas e Eletrônicos (IEEE). Foi Professor Visitante no Departamento de Engenharia Elétrica e de Computação, Universidade de Toronto, entre julho e agosto de 1997 e em abril de 2003. Revisor da revista IEEE Transactions on Communications. Revisor de diversas revistas científicas do IEEE, SBrT, SBMO e Kluwer. Tem sido, por muitos anos, membro do Comitê Técnico ou revisor da IEEE Globecom, da IEEE ICC, da IEEE WCNC, da IEEE PIMRC, IEEE WPMC, SBMO, IMOC, IEEE WS SPAWC, IEEE/IFIP ICT, IWT e do Simpósio da SBrT. Foi membro do International Advisory Committee do PIMRC'95 XV SBT, em 1997. Foi Coordenador Técnico do ITS, em 2002.
Foi membro da Comissão Organizadora do VI SBT, em 1988. Marcelo S. Alencar é membro da CBC 2 da Anatel. Membro da Câmara Técnica Setorial de Desenvolvimento Tecnológico das Telecomunicações, da Finep. Presidente do Instituto de Estudos Avançados em Comunicações (Iecom). Coordenador Geral do Grupo de Telecomunicações (Camada Física) do SBTVD. Tem sua biografia incluída nas publicações Who's Who in the World, Who's Who in Science and Engineering, publicado por Marquis Who's Who, New Providence, EUA, e Outstanding People of the 21st Century, publicado pelo International Biographical Centre, Cambridge, Inglaterra, e recebeu um patrocínio (Grant) da Fundação IEEE. Foi um dos agraciados com a homenagem pelos 20 anos da SBrT. Marcelo S. Alencar é autor dos livros Telefonia Digital (Quarta Edição) e Sistemas de Comunicações, pela Editora Érica Ltda, autor do livro Princípios de Comunicações, pela Editora Universitária, UFPB, autor do ensaio Historical Evolution of Telecommunications in Brazil, pela IEEE Foundation, é um dos autores do livro Communications, Information and Network Security, pela Kluwer Academic Publishers, e articulista do Jornal do Commercio On Line, de Recife, assinando a coluna Difusão, de divulgação científica, desde abril de 2000 . 